\title{
The Application research of Virtual cloud desktop on College computer laboratory management
}

\author{
Shigang Wang ${ }^{1}$ \\ ${ }^{1}$ Wuhan City Polytechnic, Wuhan, Hubei Province, China
}

Keywords: The virtual desktop cloud, Colleges and universities, the computer laboratory, Laboratory management.

\begin{abstract}
Computer network technology is developing rapidly, the thorough change the way people live, work, and at the same time brings convenience to people serious problem of information security. Specific to the computer lab management performance is more obvious, so in reality there is a need to be computer laboratory management research. Based on this, the author in this study combined with practical work experience, and analyze the present situation of computer laboratory management and cloud desktop is introduced in detail in the application of the computer laboratory management.
\end{abstract}

\section{Introduction}

With the rapid development of information technology and computing machine in all the fields should be used extensively, every professional in institutions of higher learning established close ties with the computer, in a computing machine on chamber training and exercise the students comprehensive ability has become the special industry in colleges and universities the division teaching or lack of an important part [1]. Meter is the construction of the machine room on also heavy to work for this into colleges and universities. But so far, the meter computer room on the construction and management of side surfaces are still exist many problem, such as how to apply new technology developments, compose built for more efficient use of computing machine real LABS and the experimental teaching system, the value we deeply research. Cloud computing era to come for real in laboratory construction and management of colleges and universities provide a new thinking way.

\section{Main problems of College computer laboratory management in the daily usage}

Large amount of system and the application daily maintenance. Because of the school computer room PC number is larger, when teaching software, update the application or operating system upgrade patch, etc and virus attack requires administrators to spend a lot of time and effort to rebuild the teaching environment, reduces the network management of maintenance and management efficiency, and affects the normal teaching [1].

The teaching and controlling of continuity is bad. To reduce a certain amount of maintenance and wide spread use of "recovery", in order to prevent the students can install the software administrator deploy teaching environment, leads to the courseware of the teachers and students homework can't save, in the last lesson of unfinished experiments also is not completed until next class to continue, will produce the problem such as radio desktop can't fully control in place.

The lack of sophistication in teaching. Because of college professional and different materials, software variety, large quantity, the cost is high, cause the purchasing cycle is long, lead to the use of teaching software, may be behind the mainstream of society and market software at least 1-2 years [2]. And some software deployment complexity is higher, special requirements for hardware and network environment, make them unable to use rapid deployment in school. So how to speed up the new technology, the mainstream software applications and rapid deployment of online is more concern for colleges and universities.

IT asset management is not perfect. Distribution in every classroom of the PC configuration statistics and protect the hardware security relatively trouble, cause more easily remember, or to register and actual configuration is not consistent, and so on and so forth. 
High capital investment and management maintenance costs. The construction of colleges and universities due to various reasons, the computer classroom investment co., LTD., coupled with the rapid development of IT technology, generally 3 to 5 years by the machine needs to be updated out a group of poor performance. And this kind of repeated purchasing, selection and purchasing, to eliminate, in fact, a kind of serious waste of resources [2]. And desktop computers in terms of its energy consumption than high, conflict with the concept of low carbon environmental protection. So how to guarantee under the premise of teaching environment, decrease the cost of funding and management of campus computer maintenance is also concern in colleges and universities have been.

\section{Cloud computing and cloud desktop technology}

Cloud computing is a hot topic in recent years, information technology and its development trend. There are many definition of cloud computing. That is cloud computing, can be found at least 100 explanations [3]. Widely accepted at the present stage is the U. S. national institute of standards and technology definition: cloud computing is a kind of pay by usage pattern, this pattern provides available, convenient, on-demand network access, enter the configurable computing resources Shared pool (resources including network, servers, storage, applications, services), these resources can be quick to provide, just in the management of the very few and or interactions with the service provider with few, shown as Fig. 1. The application of cloud computing in order to reduce costs, improve production efficiency and business systems brought immeasurable value rapid deployment.

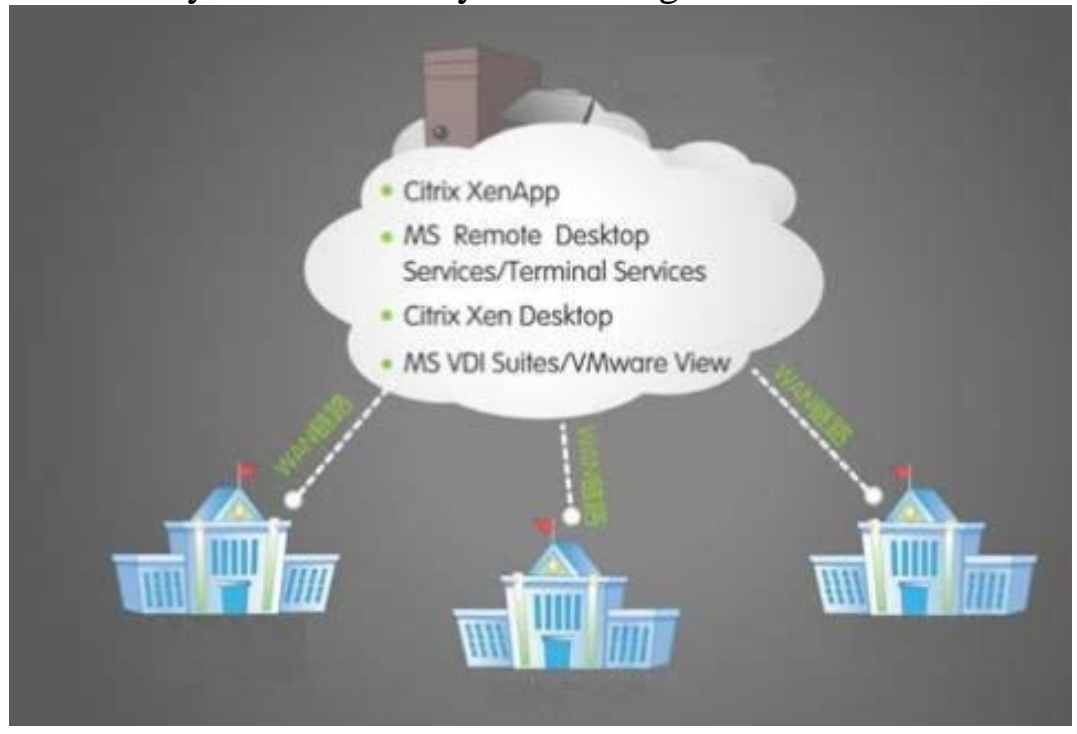

Fig. 1 Virtual cloud diagram

Cloud desktop is a typical application of cloud computing, cloud computing based on distributed storage technology, integrated essence of Internet applications, relying on high encryption algorithm, to all levels on the Internet users with the most simple, the most abundant and the safest, most thoughtful service [3]. Desktop virtualization, its core technology is a new idea of solutions and infrastructure, its principle is on the server side for each user dedicated virtual machine and deployment in which the user required operating system and a variety of applications, and then through the desktop display agreement will complete virtual machine delivered to the remote desktop users. So we can put all the desktop virtual machine in a data center hosting and unified management, at the same time, the user can get full use of PC. Its value lies in information security, convenient maintenance, energy saving, rapid deployment, equipment replacement frequency is low, outside the office is convenient, etc.

\section{Computer lab network security features}

Authenticity and reliability. Authenticity this characteristic can be divided into two sides, this paper focuses on the user, is real and effective authorized user identity information, mainly to avoid an impostor situation appear in the network environment, to identify the authenticity of the user 
identity information is particularly important; Is the network system reliability in certain situations on a particular task characteristics, is also the basic goal of building computer network security defense technology [3].

Confidentiality and integrity. The computer network information security and protection, confidentiality and integrity of the important task are to ensure the safe operation of computer network [4]. In the operation of the network to ensure that confidential information not be leaked to unauthorized users is the confidentiality; Ensure that will not be malicious tampering with important information in the operation of the computer network, network information storage transmission at the same time don't appear in the process of change, damage and loss.

Controlled with a possessive. Controlled to ensure the network spread bad information will not be with transmission, a wide range of adverse effects, influence the network running environment of security and stability [4]; A possessive is computer network information security system to the authorized user of the special performance, is embodied in two aspects: to provide information service to authorized users, diversity and sexual needs at any time.

\section{The application of cloud desktop computer laboratory management technology}

Part mainly on analyzing the characteristics of the computer laboratory management in network security, for the next paper, analysis of cloud desktop technology application in the computer room management, from the design thought, structure system, and analyzes the specific application.

Design application. First according to the curriculum to make virtual machine, and then install the operating system in virtual machine, according to the required to install the corresponding teaching application and management of plug-in, the encapsulated into a virtual machine image template, according to the course name again. If different need to apply to the same or similar teaching application, can after considering it's merged into the same virtual machine, and then to encapsulate it into image template, when naming comments should be clear, this can largely reduce the saving storage space [4].

System architecture. Combined with cloud desktop computer room management system and working principle of cloud desktop, can according to the analysis and control scheme of the hierarchical it into the client layer [4]. Cloud host layer and management layer. The client layer is mainly aimed at different studio teaching environment design cloud computer terminals; Cloud host layer is in accordance with the requirements of curriculum and teaching building highly qualified software image, again through the host access client layer; Management is the deployment of related applications and centralized management of a management platform, also can have the effect of safety monitoring of the whole system.

Personalized teaching scene delivery and optimization. Traditional physical PC build computer classroom, classified management in the operating system installation and software would be more complicated, low efficiency, and poor user experience. If a single machine at the same time need to install Windows, Linux, Mac OS operating system, the implementation process is relatively complex and high failure rate [5]. At the same time, due to the operating system can't install more, only according to the application of classification or discipline direction, set up two or three operating system, and applies all belong to the category of software all installed under the same operating system. This kind of planning method, which leads to the operating system, large start slow, many software, users choose trivial, hidden danger of many problems, such as software conflict. So, how to realize the personalized teaching scene delivery and optimization, is the important aspect of prominent cloud desktop technology advantage, is also a need to consider in the process of system design and implementation of the key problems.

Through the cloud desktop technology, can be on the server cluster virtual hardware layer, almost all types of operating system installation, and will not interfere with each other. Because use stateless desktop instant deployment, the administrator can establish personalized for each teacher teaching desktop, need only install this term the teacher in class teaching software, the system operation speed, interface is simple [5]. Can also according to the teacher resource consumption situation of running software, the hardware configuration of personalized adjustment cloud desktop, such as CPU and 
memory resources, to achieve without the needs of the application scenario. At the same time, through the way of planning tasks, depending on the class time and classroom teachers, automatically for each classroom mount class need a desktop environment, realize automation desktop instant delivery. In addition, the cloud desktop technology, but also before class or recess in a short period of time, a rapid increase, modify, delete, application software, in order to satisfy the demands of teaching environment. The cloud desktop technology advantage, will significantly improve the teachers and students experience.

Centralized management background. Traditional way entity PC construction of multifarious work operations has been the computer room management at the core of the problem. For front end, along with the increase of the using time, more than three years of using PC, due to component aging, failure rate will increase significantly, hardware maintenance tasks [6]. While using the embedded architecture of thin client, due to the low power design and components selection, in the process of more than 5 years of use for a long time, can keep the lower frequency of a hardware failure, reduce the labor cost of hardware maintenance. In addition, the application software maintenance, occupies a lot of work time management, the administrator to update the teaching software, need to stop the use, USES the network with the way software deployment, 100 the size of the classroom, software maintenance needs at least 4 to 6 hours. And cloud desktop technology was used to construct computer classroom, because the user's desktop are dynamically generated by the template file, administrators only need to maintain a template file operation, the user can obtain need software resources, without interrupting the classroom can be used in a very short time to fulfill the requirements of users [5]. So, through the in-depth study of cloud desktop technology can be reduced from computer classroom operational difficulty, reduce maintenance workload, obtain substantial gains.

Determine the security hidden danger. When computer network failure problem during the inspection determine computer itself has no problems, problems arise out device, open the computer after the equipment to produce the source of the problem will appear on the screen [6]. Check the computer port connection is normal or not, if the port is loose to reinforcement; If it is not the problem in the outside devices, no matter what the reasons at this point you should replace the equipment, and then according to the specific situation analysis, the whole process need to be flexible use, don't be too rigid; Eliminate power failure (such as poor contact or wire short circuit), if the computer does not start normal boot, is mostly caused by the host fault [7]. Generally the abnormal problems, graphics and CMOS memory operation is not smooth; first check whether the host is normal operation, the host of fault detection, different fault, cause and treatment is not the same.

Set the virus firewall. Computer firewall filter cannot effectively guard against and viruses, to increase regularly scan files in the local network and software scanning frequency on a regular basis. The virus or antivirus software installed on the web server and the host, ensure the safety of network application. In addition, to strengthen network directory and file access restrictions, to achieve the purpose of identifying and intercept the virus. Grading firewall sort is more commonly:

1) Type. Grading the basic principle of firewall is: according to certain network security strategy, formulate the corresponding packet filtering rules. When forwarding group, according to the rules of packet filtering check [7].

2) Application proxy firewall. Its working principle is through the change of the client, the TCP connection from the trusted network relay to untreated network.

Timely response and recovery. Found malicious destruction, in network security fire prevention system should be timely response and to fight back, avoid to produce greater damage. When found after the intrusion behavior system to respond in a timely manner, protect the user's computer network security, reducing the loss of network information, increase security strength, is now the most widely used a forensics system, such as attack source tracking system [7]. Through the use of these tools to ensure the timely and effective response, against hacking, improving computer network security system; Computer network security protection impossible foolproof, so the network system security system becomes very important to recover. Recovery is a kind of make up the behavior, through the perfect backup mechanism and recoverability, high-end technology to ensure that information to 
avoid missing important information. Through effective restoration measures to control network malicious destruction, reduce the network information security risks; ensure the safety of network operation.

\section{Summary}

Widespread popularization of computer network technology, bring convenience to people at the same time also brings a series of security problems, the computer laboratory management bring new challenges, so the introduction of new technology has practical significance. In this article, analyzing the characteristics of the computer network security, to the concrete application of cloud desktop technology, ensure the quality of computer laboratory management.

\section{References}

[1] Sh. Q. Wang, Cloud desktop technology in the application of computer laboratory management, Information and computer (theory), 2016, vol. 1, pp. 32-34.

[2] Sh. X. Wang, The application of cloud computing in university computer room management, The management informationization, 2016, vol. 16, pp. 25-27.

[3] Zh. H. Wu, The applicability of the VDI and IDV in university computer laboratory analyses, Computer knowledge and technology, 2016, vol. 21, pp. 65-68. .

[4] Ch. M. Wang, Virtual desktop cloud in university research, The application of information technology and information technology, 2015, Vol. 12, pp. 28-30.

[5] W. P. Zhou, Cloud desktop in university laboratory the intelligent application of information technology and information technology, China social sciences press, 2015, Vol. 2, pp. 20-22.

[6] T. T. Feng, Desktop cloud based multimedia computer laboratory management research, Experimental technology and management, 2015, Vol. 32, pp. 164-166.

[7] J. B. Huang, Cloud computing in the university's development and construction, Network education in China, 2012, Vol. 2, pp. 13-16. . 